\title{
An Adaptive Decision Feedback Equalisation For Broadband Powerline Communication System
}

Werneld Egno Ngongi ( $\nabla$ werneld@aol.com )

Dar es Salaam Maritime Institute https://orcid.org/0000-0002-5775-6066

\section{Fortunata Kakwaya}

Dar es Salaam Maritime Institute

Justinian Anatory

Mbeya University of Science and Technology

\section{Research Article}

Keywords: Equalization Techniques, Broadband Powerline communication, Adaptive Decision Feedback, Channel Impulse Response, Channel models

Posted Date: July 13th, 2021

DOI: https://doi.org/10.21203/rs.3.rs-643190/v1

License: @ (i) This work is licensed under a Creative Commons Attribution 4.0 International License. Read Full License 


\title{
An Adaptive Decision Feedback Equalisation for broadband Powerline Communication System
}

\author{
Werneld Egno Ngongi and Fortunata Kakwaya \\ Department of Marine Engineering, Dar es Salaam Maritime Institute, P. O. Box 6727, Dar es \\ Salaam Tanzania \\ Justinian Anatory \\ Mbeya University of Science and Technology, Mbeya, Tanzania
}

\begin{abstract}
Power line networks can be used to increase accessibility of broadband communication services in developing countries. Nevertheless, power line networks are affected by stochastic channel alterations triggered by load connection and disconnection, branched line lengths, branches, etc. This impairment affects the implementation of Broadband Power Line Communication (BPLC) system. This paper therefore proposes an Adaptive Decision Feedback Equalisation (ADFE) technique to overcome the stochastic channel changes in powerline communication channels.

An appropriate power-line channel model is selected and channel impulse responses are obtained from the selected channel model. The impulse responses are obtained and used for simulation to analysing the the performance of ADFE technique. The ADFE is simulated and then results are analyzed through comparisons with other equalizers in order to examine its performance. Simulation results prove that the adaptive decision feedback equalizer performs better to overcome the effects of stochastic changes in power-line network compared to other techniques.
\end{abstract}

Keywords: Equalization Techniques; Broadband Powerline communication; Adaptive Decision Feedback; Channel Impulse Response; Channel models

\section{Declarations \\ Not applicable}

\subsection{Introduction}

Communication allows people to pass, send, receive or exchange information among them. In the past few years the world has witnessed a rapid growth of technology in communication industry such as wireless technologies, internet, etc. This rapid change of technology in communication industry has made the world to be just like a single village where people can easily communicate.

However, in developing countries the issue of communication remains to be a big problem. There are several technologies being proposed to be used for implementation of communications infrastructures such as wireless technologies, etc. However, they are much expensive to be afforded by developing countries' end users. According to PC Magazine M\&NE, it shows that power line network is the largest existing infrastructure worldwide compared to public telephone network (PSTN) [1]. Researchers have proposed power line networks (PLN) can suit to be used as communication channel which means that, power lines, apart from being used to carry electricity, the same wires can be used to carry and send information. Thus, power line networks can be used to extend communication services for developing countries [2 - 3]. This is due to fact that power line network has a good penetration in cities, municipalities, etc which dearth broadband technology and the cheapest technology to implement since electric wires are ready in place. However, power line (wires) alike other communication channels is highly affected by stochastic channel variations caused by plugging and unplugging of electrical or electronic appliances such as electrical motors, televisions, refrigerators, air conditioners, etc. An additional cause of the problem is long line lengths, branched line lengths, branches etc. This effect causes the transfer function of the channel to vary with time. Since the change (vary) of the transfer function is random, hence stochastic channel changing in power line communication network (PLC) may perhaps result into loosing or distortion of data. Thus, appropriate measures are required to overthrow the problem so as to enhance the implementation of BPLC. 
In response to this; Researchers Jones and Sistanizadeh presented channel equalization technique for very high speed digital data communication [4]. The performance of the equalizer was based on the application of decision feedback equalizer (DFE) which used a fractional space equalizer (FSE). The advantage of FSE was dominance performance that it offers for an arbitrary slicer sampling phase. Drajic and others proposed adaptive channel equalization technique to suppress multiple access interference (MAI) problems in wideband code division multiple accesses (WCDMA) downlink [5]. In this method, the conventional rake receiver is first defined followed by chip level equalizer known as common pilot channel.

Rice and Satorius proposed equalisation technique which was intended for multipath mitigation in flight telemetry [6]. Knowledge-Based Adaptive Channel Equalization is another method proposed by Huihua and others [7]. This method envisioned to resolve the problem of inter-symbol interference (ISI) in gradually varying channels. In 2002, Singer and others studied about turbo equalization approach to coded data transmission over channels with intersymbol interference [8]. They presented algorithms for turbo equalization, which are applied to coded data transmission over ISI channels. Eynard and Laot presented a blind Self - Adaptive Decision Feedback Equalizer (SA-DFE). The equalizer switches to a linear recursive equalizer when the channel is very dispersive and to a decision-directed DFE when the output of the equalizer presents reliable decisions. So their study was intended on giving details about the two structures of the SA-DFE and justifies why these structures are relevant.

Degardin and others proposed a semi-blind equalization algorithm, the algorithm associated with channel state change detection to handle variations in the transfer function [9]. The algorithm was designed to suit transmission on indoor power line. This approach serves as extension of blind estimation.

Chuah (2007) proposed Adaptive Robust Turbo Equalization for PLC which acts as inner decoder and channel decoder acts as the outer decoder and it is called turbo equalizer [10]. The idea of turbo equalization lies in the exchange of soft information between the equalizer and the decoder. The turbo equalizer needs knowledge of Channel Impulse Response (CIR) to perform data estimation. Due to time-varying characteristics of power line channels, the CIR needs to be constantly estimated. Xie and Li proposed a new precoded turbo equalization scheme for power-line communication channel [11]. The scheme transforms the non-recursive ISI channel to one that is recursive by precoding it with an appropriate rate recursive precoder, hence enabling the renowned interleaving gain.

A real-time Field-Programmable Gate Array (FPGA-based) architecture designed to implement channel estimation and equalization in broadband PLC has been proposed [12]. The approach is based on Orthogonal Frequency Division Multiplexing modulation (Wavelet-OFDM), also known as Filter Bank Multi-Carrier (FBMC), where cross-correlation techniques have been applied on the Zadoff-Chu sequences used as pilot signals or preambles. Many other studies regarding equalization of PLC have been presented in [17 - 27].

From above paragraph, it has been noted that, only few researches concerning equalization in PLC have been done so far. Therefore more researches need to be conducted in this area in order to address properly the problem of equalization.

Therefore this paper presents adaptive decision feedback equalization techniques for broadband power line communication. Dissimilar power line channel models have been studied, however in this paper; the one presented by Anatory and others is used [13]. This model is suitable for a transmission line with multiple branches at a single node. The performance of the adaptive decision feedback equalizer is compared to blind and adaptive linear equalizers based on Based on Symbol Error Rate (SER) and Signal to Noise Ratio (SNR).

finally, this paper is divided into four sections were section one gives introduction, section two presents channel models, section three presents simulation and analysis of the equalizer and last section gives conclusion.

\subsection{POWER LINE CHANNEL MODELS}

\subsection{Characteristic of Power line channel}

This section presents power-line channel model used in this paper. The use of power line for communication presents a number of technically difficult challenges. In addition to large attenuation, the power line cable network is one of the most electrically contaminated environments [14]. PLNs have been assembled with a variety of materials and cross sections are joined almost at random. This means that the inductive reactance along a wire itself will 
render a wide range of characteristic impedances at different points in the networks. Again, the PLN terminal impedance varies both at communication signal frequencies and with the time of day as the network's electrical load pattern varies [14]. All these contribute to stochastic channel changing behavior of the PLC channel. Hence the transfer characteristic (transfer function), $H(f)$ of the channel is varied with respect to time.

\subsection{Power-line Channel Models}

The modeling of the PLC channel is of fundamental importance, since the quality of the transmission is highly influenced by the characteristics of the channel itself [15]. For investigating the performance of power line, a number of channel models for PLC have been proposed by researchers. However, in this paper a PLC channel model proposed by Anatory is used [13]. This model is suitable for a transmission line with multiple branches at a single node. The generalized channel transfer function is as represented as:

$$
\begin{aligned}
& H_{m}(f)=\sum_{M=1}^{L} \sum_{r=1}^{r} T_{L r} \alpha_{m r} H_{m r}(f) \quad r \neq m \\
& \alpha_{m r}=P_{L r}^{M-1} \rho_{r m}^{M-1} e^{-\gamma_{r}\left(2(M-1) \lambda_{r}\right)} \\
& P_{L r}=\left\{\begin{array}{cc}
\rho_{S} & r=1(\text { source }) \\
\rho_{L r} & \text { otherwise }
\end{array}\right.
\end{aligned}
$$

where, $N$ is the total number of branches connected at any node and terminated in any arbitrary load. $n, m, M$, $H_{m r}(f)$ and $T_{L m}$ represent any branch number, any referenced (terminated) load, number of reflections (with total $L$ number of reflections), transfer function between line $r$ and referenced load $\mathrm{m}$, transmission factor at the referenced load $m$, respectively. With these the signal contribution factor $\alpha_{m r}$ is given by (2), where $\rho_{r m}$ is the reflection factor at any node between line $\boldsymbol{r}$ to the referenced load $\boldsymbol{m}, \gamma_{r}$ is the propagation constant of line $\boldsymbol{n}$ that has line length $\lambda_{r}$. All terminal reflection factors $P_{L r}$ in general are given by (3), except at source where $\rho_{L 1}=\rho_{S}$, is the source reflection factor. Also the output referenced voltage $V_{m}(f)$ in frequency domain is given in [13]:

$$
V_{m}(f)=H_{m}(f)\left(\frac{Z_{L r}}{Z_{L r}+Z_{S}}\right) V_{S}
$$

where $Z_{S}$ is the source characteristic impedance, $Z_{L r}$ is characteristic load impedance, $V_{S}$ is the source voltage and $H_{m}(f)$ is the channel transfer function. The inductance, capacitance, resistance and conductance are calculated [13] as in (5), (6), (7) and (8):

$$
\begin{aligned}
& L=\frac{\mu}{\pi} \cosh ^{-1}\left(\frac{D}{2 a}\right) \quad[H] \\
& C=\frac{\pi \varepsilon}{\cosh ^{-1}(D / 2 a)} \quad[F / M] \\
& R=\frac{1}{\pi a} \sqrt{\frac{\pi f \mu}{\sigma}} \quad[\Omega] \\
& G=2 \pi f C \tan \delta
\end{aligned}
$$

The parameter $\mu$ is permeability in free space, $D$ is the cable diameter, $a$ is radius of conductor, $\varepsilon$ is permittivity in free space, $\delta$ is the conductivity of material, and $\sigma$ is the depth factor. The characteristic impedance is calculated as: 


$$
\begin{aligned}
& Z=\sqrt{\frac{R+j \omega L}{G+j \omega C}} \\
& \gamma=\alpha+j \beta=\sqrt{(R+j \omega L)(G+j \omega C)}
\end{aligned}
$$

Since PLC uses overhead transmission lines and the separation between two lines is free space, the conductance is assumed to be zero [13]. The propagation constant is calculated as in (10), where $\alpha$ is the attenuation constant and $\beta$ is the phase factor. With one branch PLC network the following parameters are used according to Anatory and others (Anatory et al, 2007). From Fig. 3-2, $l_{1}=60 \mathrm{~m}$ is the length from distribution transformers to first interconnection, $l_{2}=10 \mathrm{~m}$ is the length of first interconnection cable and $l_{3}=60 \mathrm{~m}$ is the length of second interconnection cable. $L_{1}=3.2735 \times 10^{-7} \mathrm{mH}$ and $C_{1}=2.7191 \times 10^{-10} \mu \mathrm{F}$ where $L_{1}$ and $C_{1}$ are inductance and capacitance of the first interconnection respectively; $L_{2}=4.5179 \times 10^{-7} \mathrm{mH}$ and $C_{2}=1.9702 \times 10^{-10} \mu \mathrm{F}$, where $L_{2}$ and $C_{2}$ are inductance and capacitance of second interconnection respectively; $C_{3}=2.7191 \times 10^{-10}$ $\mu \mathrm{F}$ and $L_{3}=3.2735 \times 10^{-7} \mathrm{mH}$ where $C_{3}$ and $L_{3}$ are capacitance and inductance of third interconnection. $L_{4}=L_{2}$ and $C_{4}=C_{2}$. from Fig. 1, the transfer function of the network is taken between point A and $\mathrm{B}$.

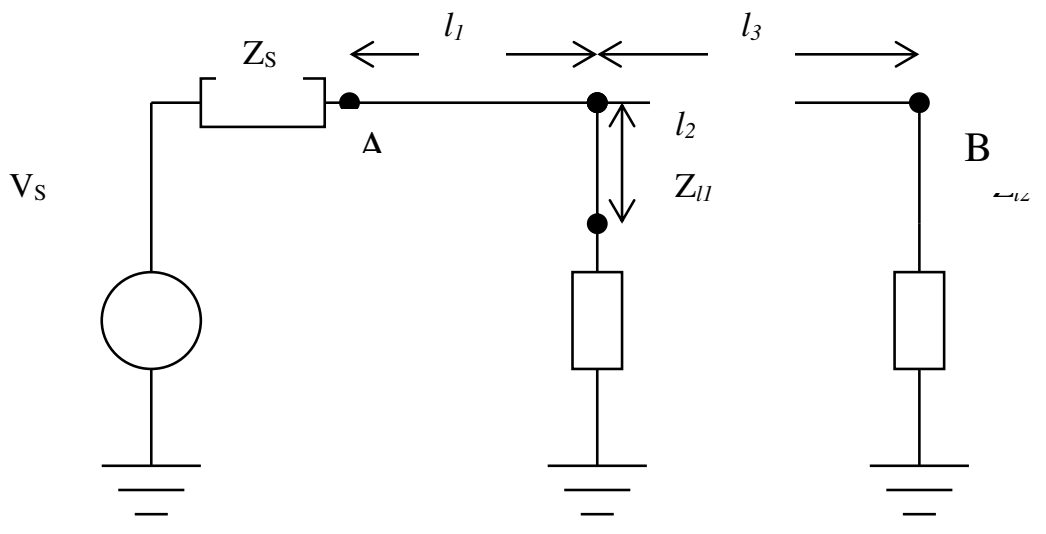

Fig. 1: PLC channel with a Single branch

The channel response for the network is as shown in Fig. 2. From Fig. 2 the values of the channel impulse response can be obtained. 


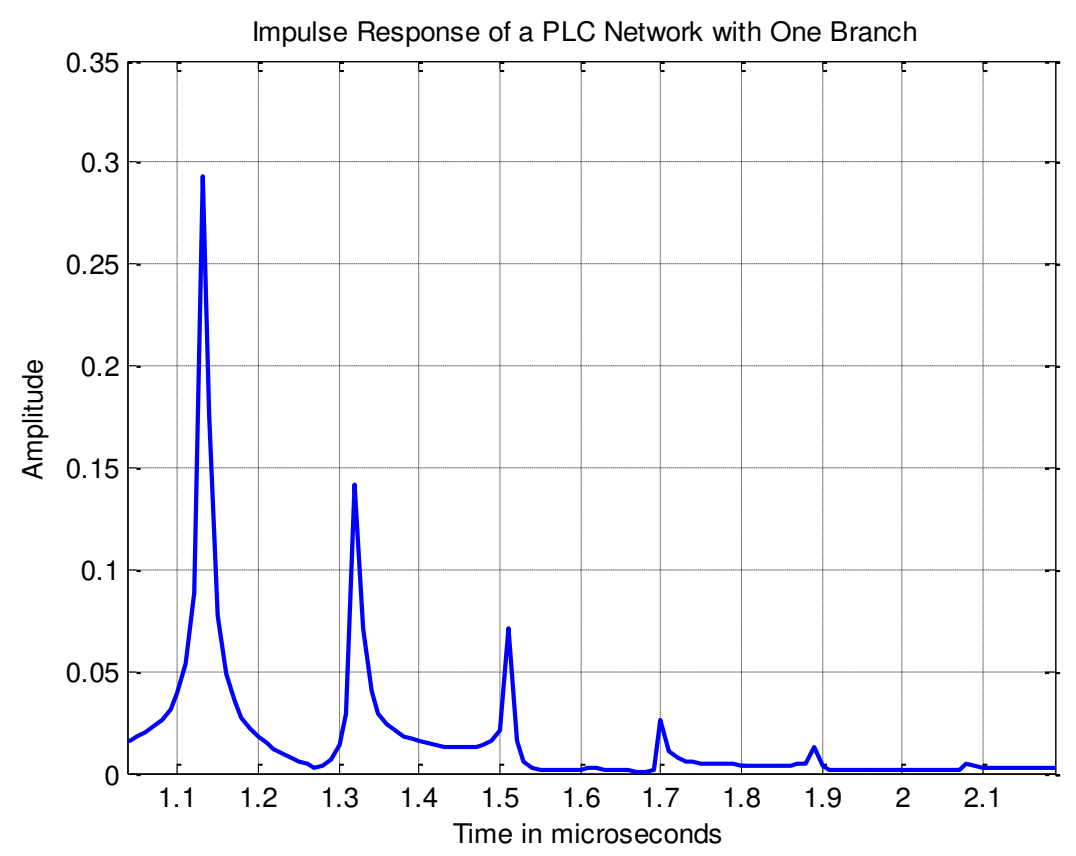

Fig. 2: Channel Impulse Response for PLC channel with Single Branch

For two branches network, the parameters are as follows (refer Fig. 3): $l_{1}=40 \mathrm{~m}$ is the length from distribution transformers to first interconnection, $\mathrm{m} l_{2}=10$ is the length of first interconnection cable and $l_{3}=40 \mathrm{~m}$ is the length between first to second interconnection cables, $l_{4}=10 \mathrm{~m}$ is the length of second interconnection and $l_{5}=40 \mathrm{~m}$ is the length of fourth interconnection. The simulation results of impulse responses are shown in Fig. 4. Again the transfer function is taken between point $\mathrm{A}$ and $\mathrm{B}$.

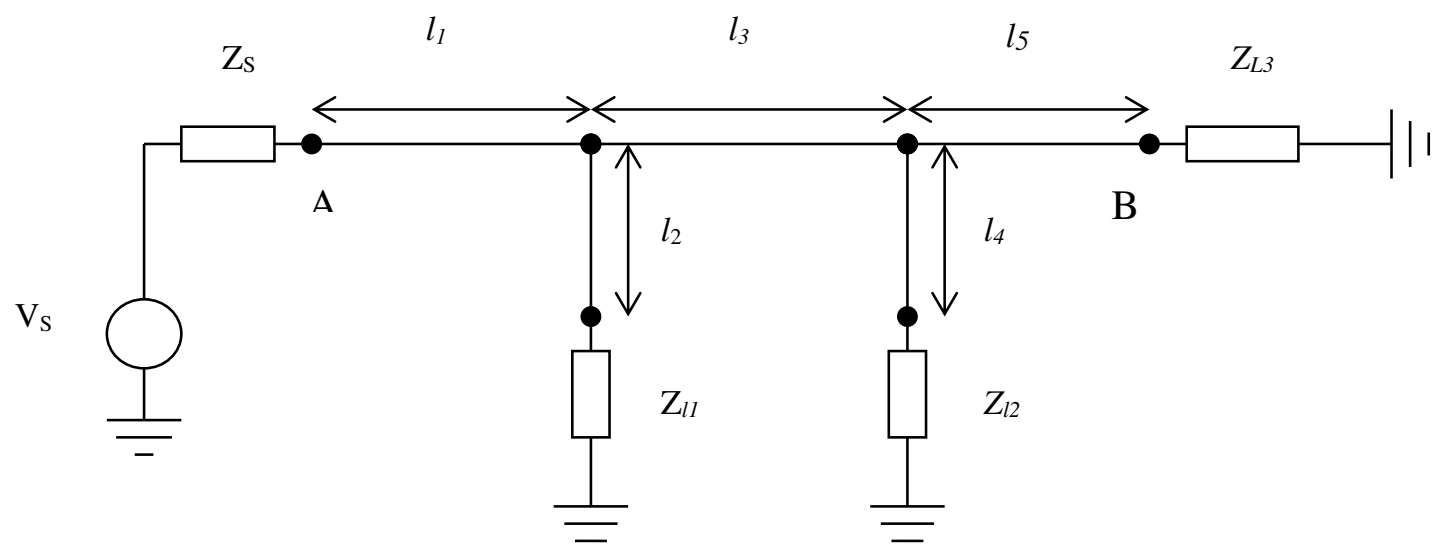

Fig. 3: PLC channel with two cascaded branches 


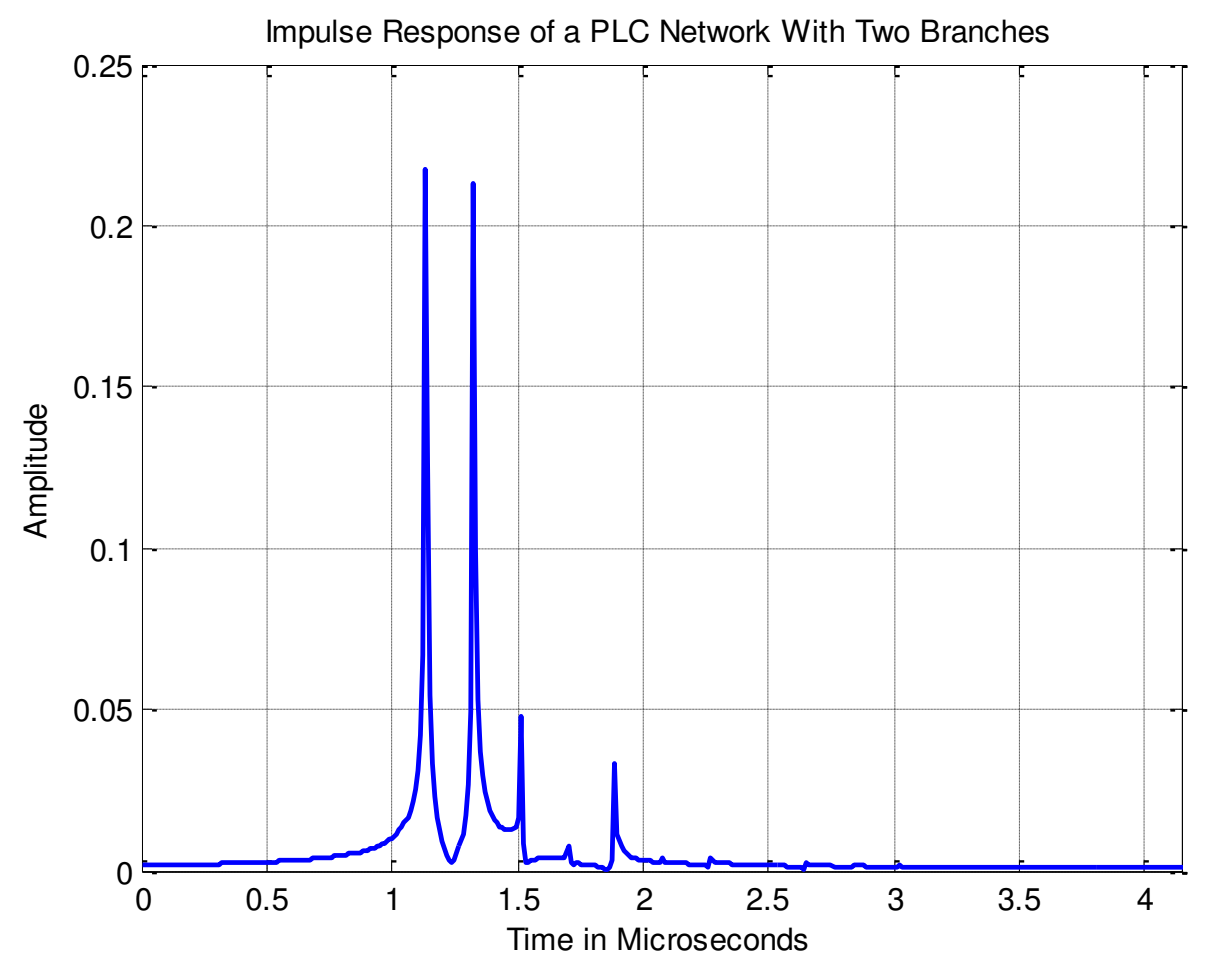

Fig. 4: Channel Impulse Response for PLC channel with two cascaded Branches

The Channel Impulse Response (CIR) parameters obtained from this section are used in simulation of to examine the performance of adaptive decision feedback equalizer.

\subsection{Simulation and analysis of Adaptive Decision-Feedback Equalizer}

This section presents the simulation of an Adaptive Decision feedback adaptive equalizer as shown in Fig. 5 and the PLC channel is applied through it [16]. Data symbols $\{s(i)\}$ are transmitted through the channel and the output sequence is measured in presence of additive white noise, $\{v(i)\}$ and interference caused by channel itself. The noisy output of the channel is denoted by $u(i)$ is fed into feedforward filter with 20 taps, as indicated in Fig. 5 [16]. Then the output of the decision device is feed into feedback filter with 15 taps, this filter works in conjunction with feedforward filter in order to supply the decision device with an estimate $\{\hat{s}(i-\Delta)\}$.

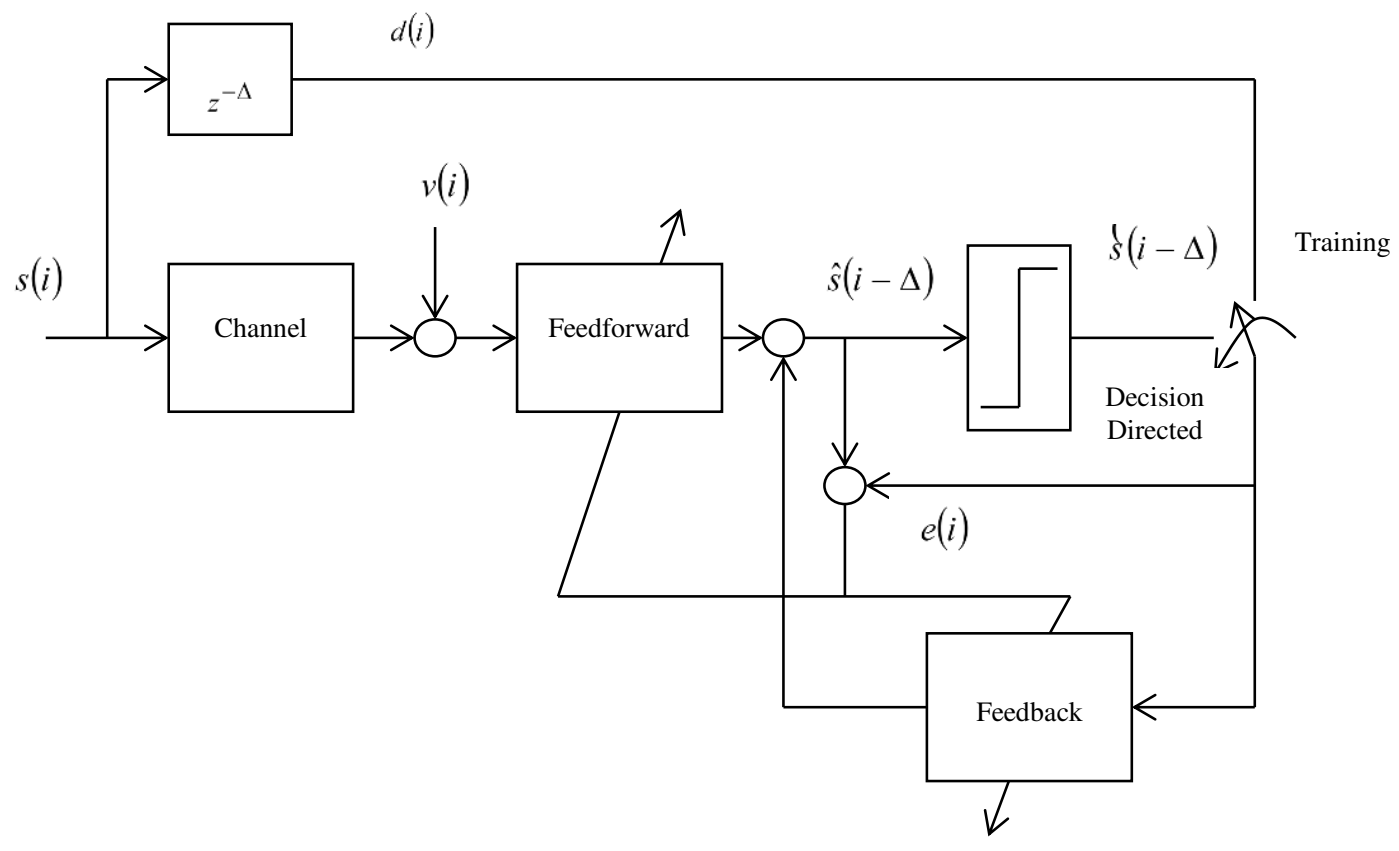


Fig. 5: ADFE simulation model. Both in decision modes of operation are shown: training decision directedoperation

During training, delayed symbols are used as reference sequence. At each time instant $i$, the symbol $d(i)=s(i-\Delta)$ is compared with the output of the adaptive filter, $\hat{s}(i-\Delta)$ (which the input to decision device), and the error signal, $e(i)=d(i)-u_{i} w_{i-1}$, is generated. The error is then used to adjust the coefficients of the feedback and feedforward filters.
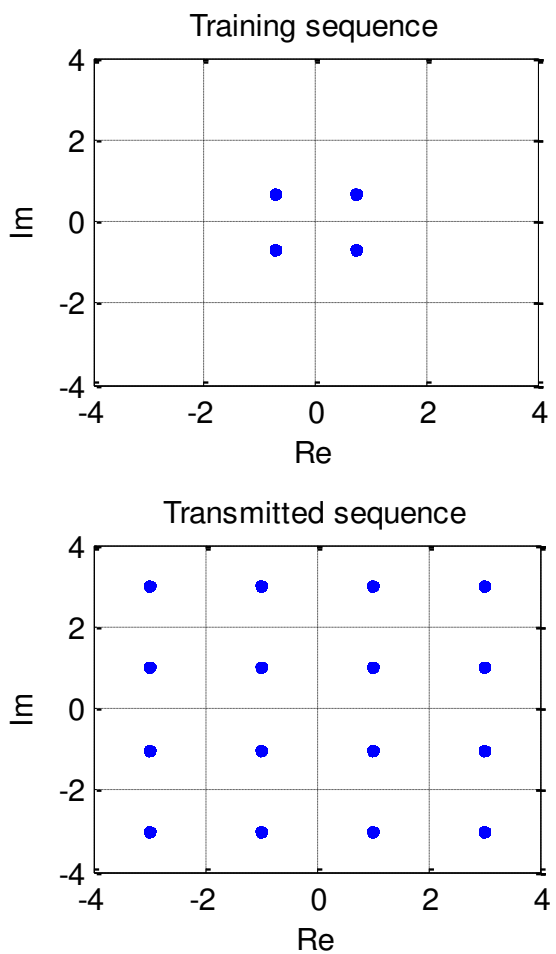

Fig. 6: Simulation Results for ADFE with Training Sequence and Transmitted Sequence

Fig. 7 and Fig. 8 below shows simulation results for PLC channel with one branch and two networks respectively. In both cases, the received signal is corrupted with ISI, and then the received signal is equalized using ADFE. The results show that, with ADFE, the equalized sequence seemed to be equalized closer to the original signal. Thus, based on the scattered diagram it can be observed that, ADFE has removed considerable amount of ISI. 

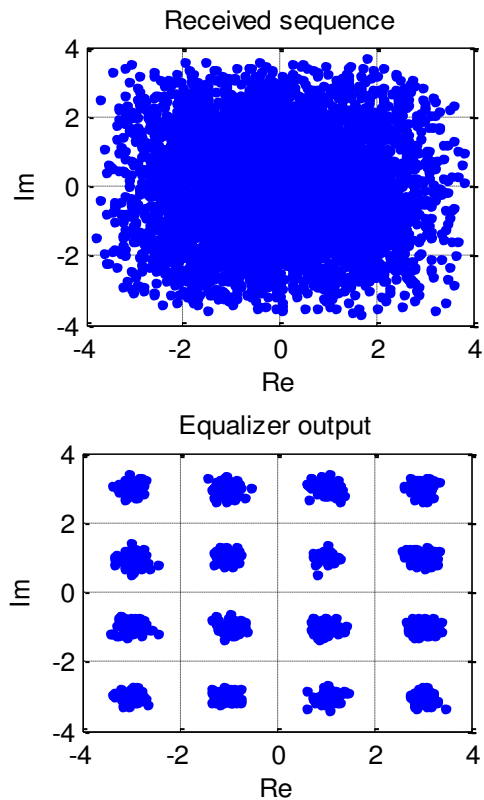

Fig. 7: Simulation Results of a PLC channel with one branch
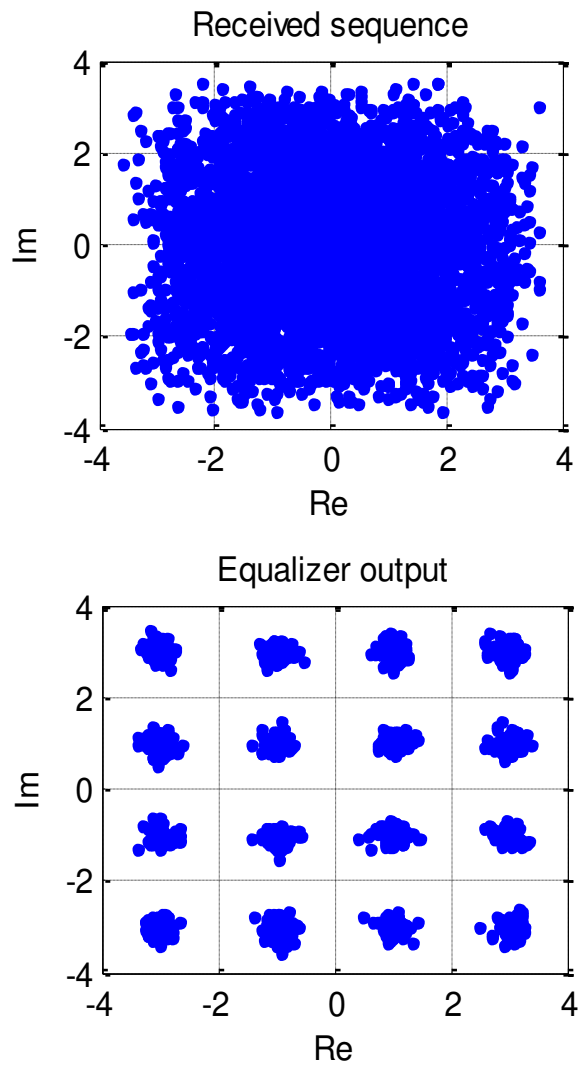

Fig. 8: Showing Simulation Results for a PLC network with two branches equalized using ADFE

\subsection{Simulation based on Probability of SER versus SNR}

This section presents comparative study of adaptive decision feedback equalizer with other equalization techniques based on Symbol Error Rate (SER) and Signal to Noise Ratio (SNR), the results for simulations are shown in Fig. 9 
and Fig. 10. Fig. 9 shows a plot of SER vs SNR for three equalizers when a PLC network with one branch is used. it can be observed from the simulation results that, the values of SER for the three equalizers decrease with increase in SNR $\left(E_{0} / N_{0}\right)$ values. But the way the SER decreases is reasonably different from each other. The two equalizers (blind equalizer and adaptive linear equalizer (ALE)) shows poor result compared to ADFE. Therefore based on the result of Fig. 9, it shows that ADFE is the best equalization technique to be employed with PLC channels.

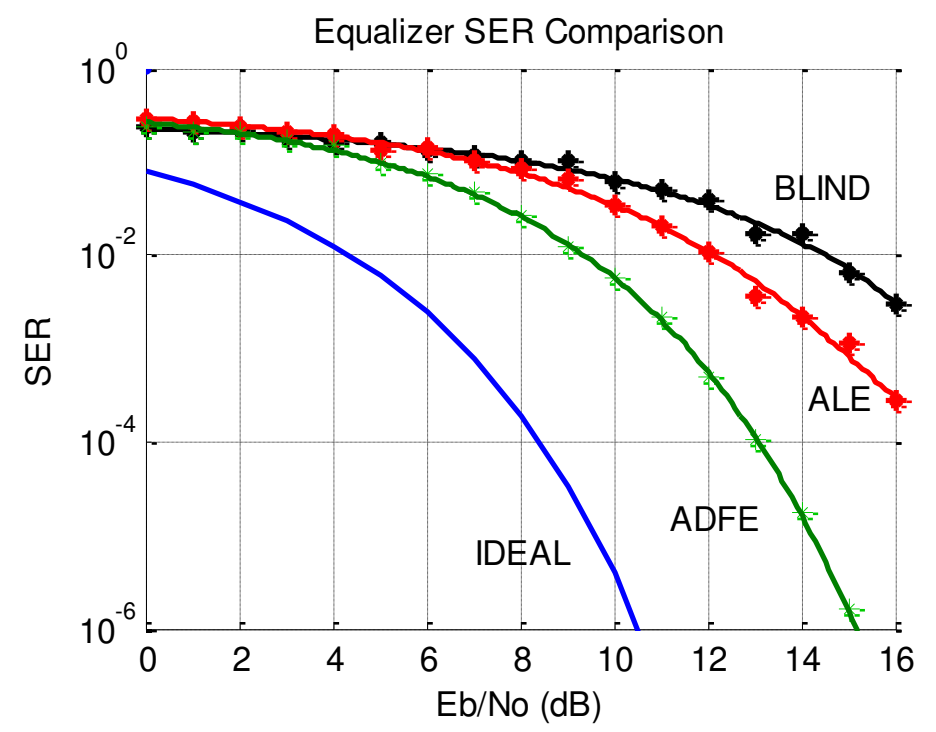

Fig. 9: A plot of SER vs SNR for a PLC network with one branch

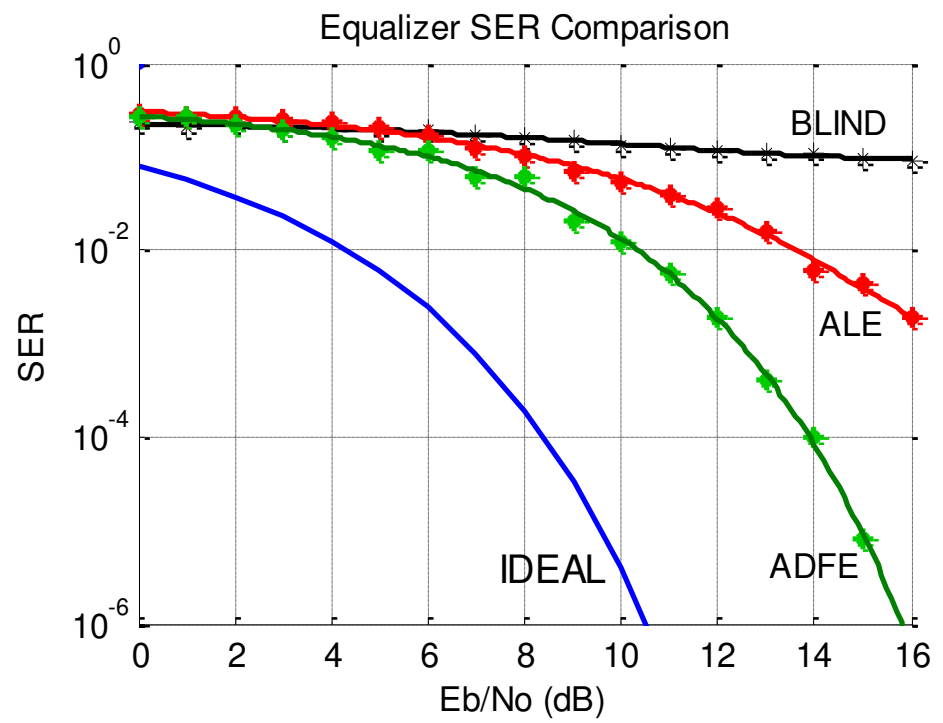

Fig. 10: A plot of SER vs SNR for a PLC network with two cascaded branches

Fig. 10 shows a plot of SER vs SNR for three equalizers when a PLC network with two cascaded branches is used. Comparing simulation results of the three equalizers given in Fig. 10, again it shows that, values of SER for all three equalizers decrease with increase in SNR $\left(\mathrm{E}_{\mathrm{O}} / \mathrm{N}_{\mathrm{O}}\right)$ values. From Fig. 10, ADFE clearly shows better performances compared to ALE and blind equalizer although ALE seemed to give better result than blind equalizer. This proves that, even for SER, ADFE is still doing better compared to ALE and blind equalizer for equalization of PLC channel. Therefore this paper proposes ADFE as the best equalization technique for PLC channel.

\subsection{Conclusion}

In this paper, an Adaptive Decision Feedback Equalisation technique has been proposed to overcome the effect of stochastic channel changes in powerline communication systems. Appropriate power-line channel model was 
selected and channel impulse responses are obtained from the selected channel model. The impulse responses are used for simulating and analysing the performance of adaptive decision feedback equalizer. Based on Symbol Error Rate (SER) and Signal to Noise Ratio (SNR), the performance of adaptive decision feedback equalizer is compared to other two equalizers. Simulation results have proved that the adaptive equalization can perform better for equalization of power-line network compared to other techniques.

\section{REFERENCES}

[1] PC Magazine M\&NE. (2004). Powerline communications: Electrifying the broadband. http://pcmag.dit.net/print.php?id=EplEpluFuVAyJBNNgf 5/4/2004. 4 May 2004.

[2] Anatory, J., Kissaka, M. M., Mvungi, N. H. (2004). Trends in Telecommunication Services Provision: Powerline Network can provide. IEEE AFRICON 2004, $602-608$.

[3] Anatory, J., Theethayi, N. (2010). Broadband Power-line Communications Systems: Theory \& Applications. WIT Press.

[4] Jones, D. C., Sistanizadeh, K. (1992). Channel equalization for Very High-Speed Digital Data Communication. IEEE Transaction on Circuits-II: Analog and Digital Signal Processing, 39(2), $116-119$.

[5] Hooli, K., Drajic, D., Tujkovic, D. (2002). Adaptive Channel Equalization in WCDMA Downlink with Turbo Coding. The $13^{\text {th }}$ IEEE International Symposium on Personal, Indoor and Mobile Radio Communications, 1442-1446.

[6] Rice, M., Satorius, E. (2005). Equalization Techniques for Multipath Mitigation in Aeronautical Telemetry, IEEE Military Communications Conference. 10.1109/MILCOM.2004.1493248.

[7] Jing, F., Yongning, C., Huihua, L. (2004). Knowledge-based Adaptive Channel Equalization. Proceedings of the $7^{\text {th }}$ International Conference on Signal Processing. 10.1109/ICOSP.2004.1452672.

[8] Singer, A. C. (2002). Turbo Equalization: Principles and New Results. IEEE Transactions on Communications, 50(5), $754-767$.

[9] Degardin, V., Lienard, M., Degauque, P. (2003). Optimisation of Equalisation Algorithm for Power Line Communication Channel. Electronics Letters, 39(5), 483 - 485.

[10] Chuah, T. C. (2007). Adaptive Robust Turbo Equalization for Power-Line Communication. IEEE Transaction on Power Delivery, 22(4), 2172 - 2179.

[11] Xie, K., Li, J. (2010). Precoded Turbo Equalizer for Power Line Communication Systems. Mathematics, Computer Science.

[12] Nombela, F., García, E., Mateos, R., Hernández, Á.(2019). Real-time architecture for channel estimation and equalization in broadband PLC. Microprocessors and Microsystems, 65, $121-135$.

[13] Anatory, J., Kissaka, M. M., Mvungi, N. H. (2007). Channel Model for Broadband Power-Line Communication. IEEE Transactions on Power Delivery, 22(1), 135-141.

[14] National Communications System. (2007). Broadband over Power Lines. Technical Information Bulletin, 14-17, http://www.comtechnologies.com.

[15] Barmada, S., Musolino, A., Raugi, M. (2006). Innovative Model for Time-Varying Power Line Communication Channel Response Evaluation. IEEE Journal on Selected Areas in Communications, 24(7), $1317-1326$.

[16] Sayed, A. H. (2003). Fundamentals of Adaptive Filtering. John Wiley and Sons, Inc.

[17] Ma, Y. H., So, P. L., Gunawan, E., Guan, Y. L. (2004). Analysis of Impulsive Noise and Multipath Effects on Broadband Power Line Communications. International Conference on Power System Technology. 10.1109/ICPST.2004.1460222.

[18] Moya, S., Hadad, M., Donato, P., Funes, M., Carrica, D. (2016). Channel estimation and equalization of broadband PLC systems: Comparison between Single Carrier and OFDM approaches. IEEE Biennial Congress of Argentina (ARGENCON).

[19] Sheikh-Hosseini, M., Molavi-Kakhki, M., Hodtani, G. A. (2012). Frequency-Domain Equalization for Orthogonal and Quasi-Orthogonal STBCs over Frequency-Selective Wireless and Power-Line Channels. Wireless Personal Communications, 71, 2445 - 2461.

[20] Ribeiro, M. V. (2005). Learning Rate Updating Methods Applied to Adaptive Fuzzy Equalizers for Broadband Power Line Communications. EURASIP Journal on Advances in Signal Processing, 16, 2592 2599. 
[21] Sheikh-Hosseini, M., Molavi, M. (2009). Single Carrier Transmission in Power Line Channels Using Time and Frequency Domain Decision Feedback Equalizations. International Journal of Tomography \& Simulation, 12(F09).

[22] Shbat, M., Ordaz-Salazar, F., González-Salas, J. S. (2017). Nonlinear Channel Equalization Approach for Microwave Communication Systems. Microwave Systems and Applications.

[23] Kit, W., Heng, W., Lim, S., Lim, H. S. (2006). An Extended Kalman Filter Based Decision Feedback Fuzzy Adaptive Equalizer for Power Line Channel. IEEE International Symposium on Power Line Communications and its Applications.

[24] Kit, W., Heng, W., Lim, S. and Lim, H. S. (2007). A robust and effective fuzzy adaptive equalizer for powerline communication channels. Neurocomputing. 71(1-3), 311-322.

[25] Seijo, M., López, G., Matanza, J., Moreno, J. I. (2016). Planning and Performance Challenges in Power Line Communications Networks for Smart Grids. International Journal of Distributed Sensor Networks.

[26] Varma, M. K., Jaffery, Z. A., Ibraheem. (2016). Advances of Broadband Power Line Communication and its application. Annual IEEE India Conference (INDICON). New Delhi, India.

[27] Ribeiro, M. V. (2004). Learning Rate Updating Methods Applied to Adaptive Fuzzy Equalizers for Broadband Power Line Communications. EURASIP Journal on Advances in Signal Processing, 2004. 